\title{
Desenvolvimento de um Modelo para Resposta Imunológica Primária Célula-mediada ${ }^{1}$
}

S. BARROZO ${ }^{2}$, Departamento de Físico-Química, Instituto de Química, UNESP, Rua Prof. Francisco Degni s/n, 14800-900 Araraquara, SP, Brasil

H.M. YANG 3 , Departamento de Matemática Aplicada, IMECC, UNICAMP, Cx.P. 6065, 13081-970 Campinas, SP, Brasil.

Resumo. Neste trabalho apresenta-se o desenvolvimento de um modelo matemático que descreve a ação do sistema imunológico adaptativo (a fim de eliminar elementos estranhos ao corpo), através das respostas humoral (produção de anticorpos pelas células B) e celular (ação de células T citotóxicas). Primeiro, mostra-se a dinâmica do crescimento do micro-organismo considerando limitação de suprimentos para sua replicação e, a seguir, a homeostasia das células do sistema imunológico. A despeito da simplicidade da dinâmica das populações de patógeno e das células, a descrição da interação de todas as populações torna-se altamente complexa. Assim, a apresentação do desenvolvimento de um modelo complexo de interação é justificada pela necessidade de sua simplificação.

\section{Introdução}

Neste trabalho apresenta-se o desenvolvimento de um modelo matemático para descrever a dinâmica da resposta imunológica humana diante de um estímulo antigênico pela introdução de um micro-organismo [5] [7] [8] [9]. A resposta do sistema imunológico consiste na eliminação de micro-organismos circulantes na corrente sanguínea (ação de anticorpos) e alojados no interior de células-alvo para se replicarem (ação de células citotóxicas). Os anticorpos são produzidos pelas células $B$ (produzidas na medula óssea) e as células $T$ (produzidas no timo) são as responsáveis pela destruição das células infectadas. Contudo, a ação coordenada e altamente específica do sistema imunológico é resultado de um processo complexo da interação entre as células $B$ e $T$ com o antígeno, além de outras células, como as apresentadoras de antígenos (macrófagos e células dendríticas, as mais importantes).

O processo de desenvolvimento de um modelo matemático envolve o estudo da dinâmica do micro-organismo invasor e das células do sistema imunológico $(B, T$ e as apresentadoras de antígenos) isoladamente; para, então, obter um cenário quantitativo da complexa interação do sistema imunológico com o parasita (antígeno).

\footnotetext{
${ }^{1}$ Apoio financeiro CNPq (Edital Universal 01/2002)

${ }^{2}$ sbarrozo@iq.unesp.br, apoio financeiro FUNDUNESP

${ }^{3}$ hyunyang@ime.unicamp.br, apoio financeiro CNPq
} 
O estudo de cada parte que atua na defesa do organismo é de suma importância, pois fundamenta as simplificações introduzidas no modelo completo (e complexo) com o propósito de se obter resultados analíticos que permitam entender a dinâmica global da interação entre parasita e sistema imunológico [3]. Assim, apresenta-se a modelagem da resposta imunológica, e, então, a obtenção do modelo simplificado.

\section{Modelando a Resposta Imunológica}

Apresenta-se a dinâmica do parasita e a dinâmica das células do sistema imunológico, aplicando-se a homeostasia, no sentido amplo, para a população de células.

\subsection{Dinâmica Microbiana}

Quando um micro-parasita invade o organismo humano, inicialmente circula pela corrente sangüínea em busca de um local apropriado para sua replicação. Este local varia de acordo com as suas características, podendo ser células (vírus e bactérias) ou regiões extra-celulares (bactérias). Durante o período em que está circulando, se detectado por alguma célula do sistema imunológico, como anticorpos, células dentríticas, macrófagos, fagócitos, sistema de complementos, dentre outras, é eliminado do organismo e seu ciclo, portanto, interrompido. Se passar por esta primeira linha de defesa, se alojará e se replicará, sendo que seus "descendentes" serão liberados na corrente sangüínea para dar continuidade ao ciclo. Parte deles serão inviabilizados por replicação defeituosa ou incapacidade de encontrar o local de replicação, ou seja, existe uma taxa de inviabilidade (mortalidade, daqui em diante) natural destes micro-organismos. Este modelo explora o caso em que os micro-organismos dependem de células-alvo para se multiplicarem, liberando muitos outros na corrente sangüínea. O mesmo princípio, porém, é válido para parasitas extra-celulares encontrarem suprimentos para replicação.

Supondo que o sistema imunológico do hospedeiro esteja incapaz de responder ao estímulo antigênico, é possível estudar a dinâmica intrínseca do parasita e, após a sua compreensão, incorporar a ação do sistema imune nesta dinâmica. Quando se considera o sistema imunológico em estado de "dormência", é de se esperar que o parasita cresça sem nenhuma resistência, inicialmente. Todavia, à medida em que sua concentração no organismo do hospedeiro aumenta, exaurem-se as célulasalvo, comprometendo, assim, a sua capacidade de manutenção. Assim, a dinâmica dos micro-parasitas pode ser descrita por meio do sistema de equações diferenciais ordinárias

$$
\left\{\begin{array}{l}
\frac{d A}{d t}=\Phi(A) H-\mu_{A} A \\
\frac{d H_{0}}{d t}=k_{H_{0}}-\mu_{H_{0}} H_{0}-\nu(A) H_{0} \\
\frac{d H}{d t}=\nu(A) H_{0}-\left(\mu_{H}+\mu_{H}^{\prime}\right) H,
\end{array}\right.
$$

onde $A, H_{0}$ e $H$ denotam as concentrações por $m^{3}$, no instante de tempo $t$, de 
antígenos, células-alvo livres e infectadas por parasita, respectivamente. As taxas per-capita de crescimento (replicação) do parasita e de ataque a células-alvo são, respectivamente $\Phi(A)$ e $\nu(A)$. As taxas per-capita de mortalidade natural dos parasitas, células-alvo livres e infectadas são, respectivamente, $\mu_{A}, \mu_{H_{0}}$ e $\mu_{H}$, enquanto $\mu_{H}^{\prime}$ é a taxa de mortalidade adicional das células $H$, induzidas pela infecção. Finalmente $k_{H_{0}}$ representa a taxa de maturação de células-alvo, um valor constante para manter constante a população de células pela homeostasia.

Quando uma célula é invadida por micro-organismo, toda a sua estrutura celular é desviada para a replicação do parasita, o que resulta na liberação de um número muito grande de parasitas e a morte celular. Assim, espera-se que a taxa de replicação seja constante, $\Phi(A)=\phi$. Contudo, a taxa de ataque a células-alvo deve depender da concentração de antígenos, uma vez que é necessária uma busca ativa por elas. Assim, o encontro com as células-alvo deve ser dificultado para baixas concentrações, porém facilitado para concentrações elevadas de antígenos, o que pode ser mimetizado por $\nu(A)=\nu A^{2}$.

Com as considerações acima, o sistema de equações (2.1) torna-se

$$
\left\{\begin{array}{l}
\frac{d A}{d t}=\phi H-\mu_{A} A \\
\frac{d H_{0}}{d t}=k_{H_{0}}-\mu_{H_{0}} H_{0}-\nu A^{2} H_{0} \\
\frac{d H}{d t}=\nu A^{2} H_{0}-\left(\mu_{H}+\mu_{H}^{\prime}\right) H
\end{array}\right.
$$

Os pontos de equilíbrio deste sistema são dados por

$$
\left\{\begin{array}{l}
P_{0}=\left(0, \frac{k_{H_{0}}}{\mu_{H_{0}}}, 0\right) \\
P_{1}=\left(\bar{A}, \overline{H_{0}}, \bar{H}\right)=\left(\bar{A}, \frac{k_{H_{0}}}{\mu_{H_{0}}+\nu \bar{A}^{2}}, \frac{\nu k_{H_{0}} \bar{A}^{2}}{\left[\mu_{H}+\mu_{H}^{\prime}\right]\left[\mu_{H_{0}}+\nu \bar{A}^{2}\right]}\right),
\end{array}\right.
$$

onde $P_{0}$ é o ponto de equilíbrio trivial, e o não-trivial $P_{1}$ (multiplicidade até dois) é dado em função de $\bar{A}$ obtido da equação de segundo grau

$$
\bar{A}^{2}-\frac{\phi k_{H_{0}}}{\mu_{A}\left(\mu_{H}+\mu_{H}^{\prime}\right)} \bar{A}+\frac{\mu_{H_{0}}}{\nu}=0,
$$

cujas soluções são

$$
\bar{A}_{ \pm}=\frac{1}{2}\left[\frac{\phi k_{H_{0}}}{\mu_{A}\left(\mu_{H}+\mu_{H}^{\prime}\right)} \pm \sqrt{1-\frac{\nu_{0}}{\nu}}\right],
$$

com $\nu_{0}=\left[4 \mu_{A}^{2}\left(\mu_{H}+\mu_{H}^{\prime}\right)^{2} \mu_{H_{0}}\right] /\left(\phi k_{H_{0}}\right)^{2}$, sendo que $\bar{A}_{+}$e $\bar{A}_{-}$são raízes (quando reais) maior e menor, respectivamente. Assim, se $\nu<\nu_{0}$, então a equação (2.4) não possui raízes reais e, conseqüentemente, o único ponto de equilíbrio (biologicamente viável) é o trivial $P_{0}$. Se $\nu=\nu_{0}$, então tem-se um único ponto de equilíbrio nãotrivial, enquanto que para $\nu>\nu_{0}$, tem-se dois pontos de equilíbrio não-triviais. 
A homeostasia da população de células é da ordem de semanas, enquanto a dinâmica de micro-organismos é de horas. De um modo geral, o sistema imunológico consegue debelar uma infecção em questão de dias, o que permite que se estude o sistema (2.2) no estado quase estacionário, ou seja, impondo $d H_{0} / d t=0$ e $d H / d t=$ 0 , e substituindo $\bar{H}_{0}$ e $\bar{H}$ (vide equação (2.3)), na equação para parasita, resultando em

$$
\frac{d A}{d t} \cong \frac{k_{1} A^{2}}{1+k_{2} A^{2}}-\mu_{A} A,
$$

onde $k_{1}=\frac{\phi k_{H_{0}} \nu}{\left(\mu_{H}+\mu_{H}^{\prime}\right) \mu_{H_{0}}}$ e $k_{2}=\frac{\nu}{\mu_{H_{0}}}$. Seja $f(A)=\frac{k_{1} A^{2}}{1+k_{2} A^{2}}$. É fácil verificar que $f(0)=0$ e $f(A) \rightarrow \frac{k_{1}}{k_{2}}$ quando $A \rightarrow+\infty$, é crescente para todo $A>0$ e tem concavidade para cima para $0<A<\sqrt{3 k_{2}} /\left(3 k_{2}\right)$ e para baixo quando $A>\sqrt{3 k_{2}} /\left(3 k_{2}\right)$, sendo limitada por $k_{1} / k_{2}$. O estudo da estabilidade das soluções de equilíbrio para a equação (2.6) mostra que $A=0$ e $\bar{A}_{+}$(raiz maior) são soluções assintoticamente estáveis, enquanto $\bar{A}_{-}$é instável (omitido aqui por ser similar ao caso da curva logística, apresentado a seguir). Isso significa que $\bar{A}_{-}$é um ponto de ruptura, usualmente denominado break point, que faz com que a proliferação, ou não, de um micro-organismo dependa fortemente da concentração inicial. Portanto, a equação aproximada para $A$ é condizente com o que se espera ocorrer na prática, pois para inoculação de parasitas em concentrações muito baixas $\left(A(0)<\bar{A}_{-}\right)$, as possibilidades de encontro com células-alvo são muito reduzidas, levando à sua eliminação. Ao contrário, se a concentração inicial for aumentada $\left(A(0)>\bar{A}_{-}\right)$, aumentam também suas chances de manutenção e, conseqüentemente, ocorre a sua instalação no hospedeiro a um nível $\bar{A}_{+}$. Note que para $k_{2}=0$ na equação $(2.6)$ tem-se uma dinâmica com quantidade ilimitada de células-alvo, ou capacidade suporte do meio infinita. Esta é uma outra possibilidade de simplificação para o modelo [2].

A suposição de estado quase estacionário para o sistema (2.2) simplifica o estudo analítico do modelo sem perder suas características essenciais, ou seja, a equação (2.6) obtida para antígenos carrega implicitamente os componentes celulares do modelo mais geral, além da infecção ser dose-dependente. Por sua vez, a equação do parasita pode ainda ser substituida por uma equação logística com efeito Allee [6], pois as dinâmicas são equivalentes. Assim, considere a equação

$$
\frac{d A}{d t}=\phi\left(1-\frac{A}{C}\right) A^{2}-\mu_{A} A
$$

representando a dinâmica populacional de parasitas, onde os parâmetros $\phi$ e $C$ são, respectivamente, a taxa intrínseca de crescimento e capacidade de suporte do meio (dado pelas condições do organismo humano). Os efeitos da competição intra-espécie, em que os indivíduos de uma mesma população competem por comida, espaço ou qualquer fonte de recursos que seja limitada, são mais pronunciados quando os encontros entre os indivíduos são mais freqüentes [4].

As soluções de equilíbrio da equação (2.7) são dadas por $\bar{A}=0$ (trivial) ou pelas raízes do polinômio de segundo grau

$$
\bar{A}^{2}-C \bar{A}+\frac{C}{r}=0
$$


onde $r=\phi / \mu_{A}$ é a capacidade reprodutiva do micro-organismo, ou seja, o número médio de replicações com sucesso gerado de uma célula-alvo infectada por um parasita. A existência e o número de equilíbrios não-triviais são facilmente determinados pela sua solução,

$$
\bar{A}_{ \pm}=\frac{1}{2}\left[C \pm \sqrt{C\left(C-\frac{4}{r}\right)}\right]
$$

sendo que: $i$ ) para baixa capacidade reprodutiva, $r<4 / C$, não se tem nenhuma raiz real positiva, tendo apenas a trivial $\bar{A}=0 ;$ ii) para capacidade reprodutiva no limiar, $r=4 / C$, tem-se, além da trivial $\bar{A}=0$, uma única raiz real positiva $\bar{A}=C / 2$; e $i$ ii $)$ para elevada capacidade reprodutiva, $r>4 / C$, tem-se, além da trivial $\bar{A}=0$, duas raízes reais positivas distintas $\bar{A}_{+}$e $\bar{A}_{-}$.

A estabilidade dos pontos de equilíbrio é dada pelo sinal de $f^{\prime}(A)=d f(A) / d A$ calculada nos pontos de equilíbrio, onde $f(A)=\frac{-\phi}{C} A^{3}+\phi A^{2}-\mu_{A} A$ e, conseqüentemente,

$$
f^{\prime}(A)=\mu_{A}\left(\frac{-3 r}{C} A^{2}+2 r A-1\right)
$$

Portanto, segue-se que: $i$ ) para baixa capacidade reprodutiva, $r<4 / C$, tem-se $f^{\prime}(0)=-\mu_{A}<0, \operatorname{logo} \bar{A}=0$ é assintoticamente estável (vale para todos os casos subseqüentes); $i i)$ para capacidade reprodutiva no limiar, $r=4 / C$, temse $f^{\prime}(C / 2)=\mu_{A}\left(\frac{-3}{C} \frac{4}{C} \frac{C^{2}}{4}+2 \frac{4}{C} \frac{C}{2}-1\right)=0$, logo nada se conclui sobre $\bar{A}=$ $C / 2$; e $i$ ii $)$ para elevada capacidade reprodutiva, $r>4 / C$, tem-se $f^{\prime}\left(\bar{A}_{-}\right)=$ $\frac{\mu_{A} r}{2}\left[\sqrt{C\left(C-\frac{4}{r}\right)}-\left(C-\frac{4}{r}\right)\right]>0$ e $f^{\prime}\left(\bar{A}_{+}\right)=\frac{-\mu_{A} r}{2}\left[\sqrt{C\left(C-\frac{4}{r}\right)}+\left(C-\frac{4}{r}\right)\right]<0$, logo $\bar{A}_{+}$e $\bar{A}_{-}$são assintoticamente estável e instável, respectivamente. Esta análise também é valida para a equação (2.6).

A análise da estabilidade assintótica do caso $i i$ ) é feita pela solução da equação (2.7), fazendo-se $r=4 / C$, a qual é dada por

$$
\int_{A(0)}^{A}\left[\frac{1}{x}-\frac{1}{x-\frac{C}{2}}+\frac{C}{2\left(x-\frac{C}{2}\right)^{2}}\right] d x=-\int_{0}^{t} d y
$$

cuja solução é

$$
\frac{\left|A-\frac{C}{2}\right|}{A} \exp \left(\frac{\frac{C}{2}}{A-\frac{C}{2}}\right)=\frac{\left|A(0)-\frac{C}{2}\right|}{A(0)} \exp \left(\frac{\frac{C}{2}}{A(0)-\frac{C}{2}}\right) \exp (t) \text {. }
$$

Note que

$$
\lim _{t \rightarrow \infty} A=\left\{\begin{array}{l}
0, \text { se } A(0)<\frac{C}{2} \\
\frac{C}{2}, \text { se } A(0)>\frac{C}{2}
\end{array}\right.
$$

o que implica que $\bar{A}=C / 2$ é uma solução de equilíbrio estável-instável. Para ficar claro, note que surgem dois ramos de raízes positivas a partir deste único ponto, 
quando $r$ aumenta. Assim, para $r>4 / C$, a solução de (2.7) é dada por

$$
\int_{A(0)}^{A}\left[\frac{1}{x}-\left(\frac{C-\bar{A}_{-}}{\bar{A}_{+}-\bar{A}_{-}}\right)\left(\frac{1}{x-\bar{A}_{-}}\right)+\left(\frac{C-\bar{A}_{+}}{\bar{A}_{+}-\bar{A}_{-}}\right)\left(\frac{1}{x-\bar{A}_{+}}\right)\right] d x=-\int_{0}^{t} d y,
$$

ou, equivalentemente, pela solução

$$
\frac{\left|A-\bar{A}_{-}\right|^{p}}{A\left|A-\bar{A}_{+}\right|^{q}}=\frac{\left|A(0)-\bar{A}_{-}\right|^{p}}{A(0)\left|A-\bar{A}_{+}\right|^{q}},
$$

onde $p=\frac{C-\bar{A}_{-}}{\bar{A}_{+}-\bar{A}_{-}}$e $q=\frac{C-\bar{A}_{+}}{\bar{A}_{+}-\bar{A}_{-}}$. Neste caso, mostra-se que

$$
\lim _{t \rightarrow \infty} A=\left\{\begin{array}{l}
0, \text { se } A(0)<\bar{A}_{-} \\
\bar{A}_{+}, \text {se } A(0)>\bar{A}_{-},
\end{array}\right.
$$

confirmando, assim, que $\bar{A}_{-}$é ponto de ruptura (instável) e $\bar{A}_{+}$é estável.

Portanto, a dinâmica populacional do micro-organismo pode ser descrita pela equação (2.7), uma aproximação do sistema de equações (2.2). Antes de estudar a dinâmica das células, note que pode-se assumir $\Phi(A)=\phi$ e $\nu(A)=\nu A$ ou $\Phi(A)=\phi A$ e $\nu(A)=\nu A$. No primeiro caso, os parasitas, mesmo diante de uma inoculação muito baixa, conseguem se estabelecer no organismo do hospedeiro, enquanto que no último caso o crescimento dos parasitas será ilimitado.

\subsection{Dinâmica celular}

O corpo humano procura manter equilíbrio, por meio de homeostasia, de todas as funções vitais. Neste aspecto, as diversas células do sistema imunológico são mantidas praticamente constantes ao longo do tempo. Assim, as células que participam da resposta imunológica, como as céluas $B, T$ e apresentadoras de antígenos, são controladas para se manterem constantes. Essa homeostasia pode ser descrita por meio de um sistema dinâmico que considera uma taxa de produção constante $k_{\bullet}$, onde • representa uma das células efetoras do sistema imunológico, e uma mortalidade per-capita $\mu_{\bullet}$, onde $\mu_{\bullet}^{-1}$ é o tempo médio de vida das células tipo •, já usado para células-alvo. Assim, a dinâmica do sistema imunológico em "repouso" é descrita por

$$
\frac{d G_{\bullet}}{d t}=k_{\bullet}-\mu_{\bullet} G_{\bullet},
$$

onde $G_{\bullet}$ representa as células $B, T$ e apresentadoras de antígenos.

A solução da equação (2.11) é dada por

$$
G_{\bullet}=G_{\bullet}(0) e^{-\mu_{\bullet} t}+\frac{k_{\bullet}}{\mu_{\bullet}}\left(1-e^{-\mu_{\bullet} t}\right),
$$

onde $G_{\bullet}(0)$ é a concentração inicial das células. Note que $G_{\bullet} \rightarrow k_{\bullet} / \mu_{\bullet}$ quando $t \rightarrow \infty$, ou seja, aproxima-se da única solução de equilíbrio da equação (2.11). 
A homeostasia age para evitar tanto a diminuição quanto o aumento das células, mantendo em equilíbrio. Isto faz com que mecanismos além da produção constante das células do sistema imunológico devem fazer parte para que ocorra rápido aumento nestas populações, e, posteriormente, leve rapidamente para valores de equilíbrio. Sendo o sistema imunológico um mecanismo de defesa bastante robusto nestes aspectos, o aumento das células é mediado por proliferação e, após debelar um invasor, retornar rapidamente aos valores de equilíbrio por apoptose (morte programada das células).

\subsection{Interação entre antígeno e sistema imunológico}

Quando um parasita invade o organismo humano, o seu sistema imunológico é rapidamente estimulado. Uma vez que os antígenos deste parasita são reconhecidos, há produção de anticorpos pelas células $B$ e destruição das células infectadas pelas células $T$ citotóxicas. Suscintamente, a estimulação do sistema imunológico considerada no modelo ocorre quando um antígeno circulante é detectado por alguma das células apresentadoras de antígenos, designadas por $C A A$, as quais são especializadas nesta tarefa, ocorrendo em maior quantidade as células dentríticas e macrófagos. Uma $C A A$ ativada pelo contato com antígeno, ao encontrar uma célula $T$ antígeno-específica, liga-se a ela ativando-a. De acordo com o tipo de antígeno, ata-se a um tipo específico de célula $T$ : associa-se a uma célula $T$ auxiliadora, $T_{a}$, no caso da necessidade de produção de anticorpos, ou a uma célula $T$ citotóxica, $T_{c}$, no caso da necessidade de destruir células infectadas pelo parasita. No primeiro caso, a célula $T_{a}$ ativada, ao encontrar uma célula $B$ que também detectou tal antígeno, ativa-a. Esta célula $B$ ativada passa por um processo de diferenciação, transformando-se em células plasma, $B_{p}$, as quais, sob efeito de citocinas produzidas pelas células $T_{a}$ ativadas, se proliferam por processo de clonagem e produzem milhares de anticorpos que são liberados na corrente sangüínea. Estes anticorpos, ao encontrarem os parasitas portadores daquele antígeno, atam-se a eles sinalizando aos macrófagos ou às células do sistema de complementos que estes parasitas devem ser eliminados do organismo. No segundo caso, a célula $T_{c}$ ativada ata-se à célula infectada e, através da liberação de citocinas especiais, provoca a morte desta última e, conseqüentemente, a do parasita. A resposta imunológica é controlada por células supressoras, e, para que as populações de células voltem a valores originais, o processo de homeostasia utiliza o recurso de apoptose, que é a morte programada das células após desempenhar sua ação na resposta imunológica.

Assim, a dinâmica da interação entre antígeno e sistema imunológico pode ser representada pelo sistema de equações diferenciais ordinárias

$$
\left\{\begin{array}{l}
\frac{d A}{d t}=\phi H-\mu_{A} A-\alpha A B_{p} \\
\frac{d H_{0}}{d t}=k_{H_{0}}-\mu_{H_{0}} H_{0}-\nu A^{2} H_{0} \\
\frac{d H}{d t}=\nu A^{2} H_{0}-\left(\mu_{H}+\mu_{H}^{\prime}\right) H-\lambda_{c} H T_{c}^{*}
\end{array}\right.
$$




$$
\begin{aligned}
& \left\{\begin{array}{l}
\frac{d B}{d t}=k_{B}-\mu_{B} B-\beta_{B} B C A A^{*} \\
\frac{d B_{a}}{d t}=\beta_{B} B C A A^{*}-\mu_{B_{a}} B_{a}-\sigma_{B} B_{a} T_{a}^{*} \\
\frac{d B_{p}}{d t}=\sigma_{B} B_{a} T_{a}^{*}-\mu_{B_{p}} B_{p}+\gamma(A) B_{p} T_{a}^{*}
\end{array}\right. \\
& \left\{\begin{array}{l}
\frac{d T_{a}}{d t}=k_{T_{a}}-\mu_{T_{a}} T_{a}-\frac{\sigma_{a} C A A^{*}}{1+\varepsilon_{a} T_{s}^{*}} T_{a} \\
\frac{d T_{a}^{*}}{d t}=\frac{\sigma_{a} C A A^{*}}{1+\varepsilon_{a} T_{s}^{*}} T_{a}-\mu_{T_{a}^{*}} T_{a}^{*}+\gamma_{a}(A) T_{a}^{*}
\end{array}\right. \\
& \left\{\begin{array}{l}
\frac{d C A A}{d t}=k_{C A A}-\mu_{C A A} C A A-\tau A C A A \\
\frac{d C A A^{*}}{d t}=\tau A C A A-\mu_{C A A^{*}} C A A^{*}+\gamma_{C A A}(A) C A A^{*}
\end{array}\right. \\
& \left\{\begin{array}{l}
\frac{d T_{s}}{d t}=k_{T_{s}}-\mu_{T_{s}} T_{s}-\sigma_{s} T_{a}^{*} T_{s} \\
\frac{d T_{s}^{*}}{d t}=\sigma_{s} T_{a}^{*} T_{s}-\mu_{T_{s}^{*}} T_{s}^{*}+\gamma_{s}(A) T_{s}^{*}
\end{array}\right. \\
& \left\{\begin{array}{l}
\frac{d T_{c}}{d t}=k_{T_{c}}-\mu_{T_{c}} T_{c}-\frac{\sigma_{c} C A A^{*} T_{a}^{*}}{1+\varepsilon_{c} T_{s}^{*}} T_{c} \\
\frac{d T_{c}^{*}}{d t}=\frac{\sigma_{c} C A A^{*} T_{a}^{*}}{1+\varepsilon_{c} T_{s}^{*}} T_{c}-\mu_{T_{c}^{*}} T_{c}^{*}+\gamma_{c}(A) T_{c}^{*},
\end{array}\right.
\end{aligned}
$$

onde tem-se as variáveis como concentrações por $\mathrm{mm}^{3}$, no instante de tempo $t$, dos patógenos e células do sistema imunológico: $A, H_{0}$ e $H$, antígenos, células-alvo livres e infectadas; $C A A$ e $C A A^{*}$, células apresentadoras de antígenos em repouso e ativadas; $B, B_{a}$ e $B_{p}$, células $B$ em repouso, ativadas e células plasma; $T_{a}$ e $T_{a}^{*}$, células $T$ auxiliadoras em repouso e ativadas; $T_{s}$ e $T_{s}^{*}$, células $T$ supressoras em repouso e ativadas (a ação destas células no modelo aparece nos denominadores); e $T_{c}$ e $T_{c}^{*}$, células $T$ citotóxicas em repouso e ativadas. Em relação aos parâmetros tem-se: $\phi, \mu_{A}$ e $\alpha$, taxas relativas aos antígenos, de replicação e de mortalidades natural e adicional devido à ação das células plasma (produzem os anticorpos); $k_{H_{0}}$, $k_{B}, k_{T_{a}}, k_{T_{s}}, k_{T_{c}}$ e $k_{C A A}$, taxas de maturação e liberação de células $H_{0}, B, T_{a}, T_{s}$, $T_{c}$ e $C A A ; \mu_{H_{0}}, \mu_{H}, \mu_{B}, \mu_{B_{a}}, \mu_{B_{p}}, \mu_{T_{a}}, \mu_{T_{a}^{*}}, \mu_{T_{s}}, \mu_{T_{s}^{*}}, \mu_{T_{c}}, \mu_{T_{c}^{*}}, \mu_{C A A}$ e $\mu_{C A A^{*}}$, taxas de mortalidade natural das células $H_{0}, H, B, B_{a}, B_{p}, T_{a}, T_{a}^{*}, T_{s}, T_{s}^{*}, T_{c}$, $T_{c}^{*}, C A A$ e $C A A^{*} ; \mu_{H}^{\prime}$, a taxa de mortalidade adicional de $H$ infectadas; $\lambda_{c}$, taxa de mortalidade das células $H$, devido à ação das células citotóxicas; $\nu$, taxa de infecção das células-alvo pelos micro-organismos; $\beta_{B}$, taxa com que as células $B$ se tornam apresentadoras de antígeno; $\sigma_{B}$, taxa com que as células $B$ apresentadoras de antígenos se diferenciam em células plasma; $\gamma, \gamma_{a}, \gamma_{s}, \gamma_{c}$ e $\gamma_{C A A}$, taxas de proliferação (clonagem) das células plasma e $T_{a}, T_{s}, T_{c}$ e $C A A$ ativadas; $\sigma_{a}, \tau, \sigma_{s}$ e $\sigma_{c}$, taxas de ativação das células $T_{a}, C A A, T_{s}$ e $T_{c}$; e $\varepsilon_{a}$ e $\varepsilon_{c}$, taxas de supressão das células $T_{a}$ e $T_{c}$. 


\section{Conclusão}

A partir do complexo sistema de interação entre parasitas e sistema imunológico, dado pela equação (2.13), busca-se possibilidades de simplificação que mantenham as características essenciais da dinâmica e, ao mesmo tempo, possibilitem algum estudo analítico do modelo. Este estudo é essencial para a compreensão desta dinâmica e a fundamentação do estudo numérico, garantindo que os resultados numéricos são consistentes e que a dinâmica não é bruscamente alterada com uma pequena alteração dos parâmetros utilizados.

Assim, para o primeiro conjunto de equações, que representa a interação do antígeno com as células-alvo, pode-se supor que o efeito da ação de anticorpos ocorre em muito maior escala do que o efeito das células citotóxicas sobre as infectadas, ou seja, $\lambda_{c} \ll \alpha$, e considera-se as células-alvo em estado quase estacionário, podendo, então, usar a equação (2.7).

Para a dinâmica das células $B$, pode-se considerar que as células em repouso e as que detectam o antígeno e se atam com as células $T$ ativadas pertençam a um mesmo compartimento, ou seja, $B^{\prime}=B+B_{a}$, o que faz com que a dinâmica destas células seja representada por

$$
\left\{\begin{array}{l}
\frac{d B^{\prime}}{d t}=k_{B^{\prime}}-\mu_{B^{\prime}} B^{\prime}-\beta B^{\prime} T_{a}^{*} \\
\frac{d B_{p}}{d t}=\beta B^{\prime} T_{a}^{*}-\mu_{B_{p}} B_{p}+\gamma B_{p} T_{a}^{*}
\end{array}\right.
$$

onde $k_{B^{\prime}}=k_{B}$ (a produção de células é a mesma, pois é regulada por homeostasia), $\mu_{B}=\mu_{B}^{\prime}$ (as células $B$ em repouso e apresentadoras de antígeno têm a mesma mortalidade, pois ainda não estão se proliferando e produzindo anticorpos, o que reduziria a vida-média) e $\beta=\sigma_{B} \frac{B_{a}}{B+B_{a}}$.

Para a dinâmica das células $T$, inicialmente considera-se as células apresentadoras de antígeno $C A A$ em estado estacionário e com isso obtém-se

$$
\left\{\begin{array}{l}
\overline{C C A}=\frac{k_{C A A}}{\mu_{C A A}+\tau A} \\
\overline{C C A}^{*}=\frac{1}{\mu_{C A A^{*}}-\gamma_{C A A}(A)} \frac{\tau k_{C A A} A}{\mu_{C A A}+\tau A} .
\end{array}\right.
$$

Considerando $\gamma_{C C A} \approx 0$ obtem-se $\overline{C C A}^{*}=\frac{\tau k_{C A A}}{\mu_{C A A^{*}-\mu_{C A A}}} \times \frac{A}{1+\frac{\tau}{\mu_{C A A}} A}$, e supondo $\frac{\tau}{\mu_{C A A}} \approx 0$ tem-se $\overline{C C A}^{*} \approx \delta^{\prime} A$, onde $\delta^{\prime}=\tau k_{C A A} /\left(\mu_{C A A^{*}}-\mu_{C A A}\right)$ é finito. Assim, a dinâmica das células $T$ auxiliadoras torna-se

$$
\left\{\begin{aligned}
\frac{d T_{a}}{d t} & =k_{T_{a}}-\mu_{T_{a}} T_{a}-\frac{\sigma_{a} \delta^{\prime}}{1+\varepsilon_{a} T_{s}^{*}(t)} A T_{a} \\
\frac{d T_{a}^{*}}{d t} & =\frac{\sigma_{a} \delta^{\prime}}{1+\varepsilon_{a} T_{s}^{*}(t)} A T_{a}-\mu_{T_{a}^{*}} T_{a}^{*}+\gamma_{a}(A) T_{a}^{*}
\end{aligned}\right.
$$

Admitindo que a ausência da supressão seja compensada pela ausência de clonagem, 
ou seja, $\varepsilon_{a} \approx \gamma_{a} \approx 0$, a dinâmica de $T_{a}$, com $\delta=\sigma_{a} \delta^{\prime}$, é dada por,

$$
\left\{\begin{aligned}
\frac{d T_{a}}{d t} & =k_{T_{a}}-\mu_{T_{a}} T_{a}-\delta A T_{a} \\
\frac{d T_{a}^{*}}{d t} & =\delta A T_{a}-\mu_{T_{a}^{*}} T_{a}^{*} .
\end{aligned}\right.
$$

Supondo que o efeito da ação de anticorpos sobressai sobre o efeito das células citotóxicas, e, como as células $T$ citotóxicas e $T$ supressoras não agem diretamente sobre os antígenos $A$, elas deixam de ser consideradas no modelo. Mais ainda, considerando equação logística com efeito Allee para a equação de antígenos e omitindo o subscrito na expressão de $T$, obtém-se o seguinte sistema para representar a dinâmica simplificada da interação antígeno-anticorpo em uma resposta imunológica primária,

$$
\left\{\begin{array}{l}
\frac{d A}{d t}=\phi\left(1-\frac{A}{C}\right) A^{2}-\mu_{A} A-\alpha A B_{p} \\
\frac{d B}{d t}=k_{B}-\mu_{B} B-\beta B T^{*} \\
\frac{d B_{p}}{d t}=\beta B T^{*}-\mu_{B_{p}} B_{p}+\gamma B_{p} T^{*} \\
\frac{d T}{d t}=k_{T}-\mu_{T} T-\delta A T \\
\frac{d T^{*}}{d t}=\delta A T-\mu_{T^{*}} T^{*}
\end{array}\right.
$$

Este sistema é estudado com detalhes em [3].

O sistema imunológico adaptativo debela uma infecção por mecanismos de ação humoral e/ou celular. Considerando ambas as respostas, obteve-se o modelo matemático dado pelo sistema de equações (2.13). Contudo, quando resposta humoral predomina (ou atua sozinha), pode-se chegar a uma simplificação do modelo, dado pela equação (3.1). As bactérias extra-celulares, não considerando outros fatores da resposta imunológica, são destruídas por ação única (no sentido de não existir ação de células citotóxicas) dos anticorpos. Em geral, ocorre uma otimização da resposta imunológica levando em consideração respostas humoral e celular, como é o caso de bactérias intra-celulares e vírus [1].

\footnotetext{
Abstract. In this paper we develop a mathematical model in order to describe both humoral and cell-mediated responses promoted by the adaptive immune system to eliminated invading parasites. We investigate the growth of the pathogen taking into account the carrying capacity and we apply the homeostasis of human body to the immune system cells. Although the dynamics of both pathogens and cells are very simple, the interaction of them is very complex. Therefore, we proposed a simplification of this complex model aiming analytical results.
}

\section{Referências}

[1] A.K. Abbas, A.H. Lichtman e J.S. Pober, "Imunologia Celular e Molecular", Revinter Ltda., $3^{a}$ Ed., Rio de Janeiro, 2000. 
[2] S. Barrozo, H.M. Yang e C.H. Dezotti, Uma abordagem matemática em imunologia, em "Matemática Aplicada à Fisiologia"(H.M. Yang, R. Sampaio e A. Sri Ranga, eds.) pp. 93-117, SBMAC, São Carlos, 2003.

[3] S. Barrozo e H.M. Yang, Mecanismos da interação antígeno-anticorpo em uma resposta primária célula T-mediada, Tendências em Matemática Aplicada e Computacional, 7, No. 1 (2006), 43-52.

[4] L. Edelstein-Keshet, "Mathematical Models in Biology", Random House, New York, 1987.

[5] C.A. Janeway Jr., How the Immune System Recognizes Invaders, Scientific American, 269 (1993), 73-79.

[6] J.D. Murray, "Mathematical Biology", Springer-Verlag, Berlin Heidelberg, 1989.

[7] M. Oprea e A.S. Perelson, Exploring the machanisms of primary antibody responses to T-cell dependent antigens, J. Theor. Biol., 181 (1996), 215-236.

[8] W.E. Paul, Infectious Diseases and the Immune System, Scientific American, 269 (1993), 91-97.

[9] A.S. Perelson e P.W. Nelson, Mathematical analysis of HIV-I dynamics in vivo, Society for Industrial and Applied Mathematics, 41 (1999), 3-44. 
